\title{
Leonhard Eulers Umgang mit langsam konvergenten Reihen
}

\author{
Walter Gautschi
}

\section{Das Basler Problem}

Eines der brennendsten mathematischen Probleme Anfang des 18. Jahrhunderts, das zwar schon im 17. Jahrhundert von Pietro Mengoli, und auch von John Wallis erwähnt, aber erst durch die fieberhaften, jedoch erfolglosen, Anstrengungen der hervorragendsten Gelehrten wie Leibniz, Stirling, de Moivre und allen Bernoullis aktuell geworden ist, bestand darin, die Summe der unendlichen Reihe

$$
1+\frac{1}{4}+\frac{1}{9}+\frac{1}{16}+\frac{1}{25}+\cdots
$$

durch bekannte Grössen auszudrücken. Ein frustrierter Jakob Bernoulli, damals wohl der geübteste Mathematiker im Umgang mit unendlichen Reihen, stellte das Anliegen [2]: „... sollte jemand das, was unseren Anstrengungen bis jetzt entgangen ist, finden und uns mitteilen, so werden wir ihm sehr dankbar sein“. Wohl infolge der grossen diesbezüglichen Bemühungen von Jakob und Johann Bernoulli ist das Problem als „Basler Problem“ in die Geschichte der Mathematik eingegangen.

Es ist bekannt, dass Euler schon 1735 das Problem gelöst, und für die fragliche Summe den Wert $\pi^{2} / 6$ angegeben hat (was ihn fast über Nacht weltberühmt gemacht hat), doch waren dieser Entdeckung - was für Euler typisch ist - numerische Rechnungen vorausgegangen. Diese sind durchaus nicht trivial, da es sich in (1) um eine sehr langsam konvergente Reihe handelt: Für eine Genauigkeit von $10^{-d}$ braucht man ungefähr $10^{d}$ Glieder der Reihe, also für sechs Dezimalstellen eine Million Glieder! Es ist daher interessant zu sehen, wie sich Euler mit dieser Schwierigkeit auseinandergesetzt hat. Wie so oft bei Euler sind aus diesem speziellen Problem Resultate hervorgegangen, die einen sehr allgemeinen und weittragenden Charakter haben. Als Beispiel hat er selbst seine Ideen auf die damals ebenso schwierige Aufgabe angewandt, die sogenannte Eulersche Konstante genau zu berechnen. 


\section{Eine erste Approximation zur Lösung des Basler Problems}

Wir schreiben

$$
s=\sum_{\nu=1}^{\infty} \frac{1}{v^{2}} .
$$

In $\$ 22$ von De summatione innumerabilium progressionum (Die Summierung einer unendlichen Reihe, E20; OI,14, S. 25-41 ${ }^{\text {; }}$, eingereicht 1731, veröffentlicht 1738) beginnt Euler mit der Integraldarstellung

$$
s=-\int_{0}^{1} \frac{\ln (1-t)}{t} \mathrm{~d} t,
$$

die man leicht durch Taylor Entwicklung von $\ln (1-t)$ und nachfolgender gliedweisen Integration bestätigen kann. Mittels der Substitution $t \mapsto 1-t$ kann man auch

$$
s=-\int_{0}^{1} \frac{\ln t}{1-t} \mathrm{~d} t
$$

schreiben. Nun zerlegt Euler das letzte Integral in zwei Teile, ein Integral von 0 bis $x$ (mit $0<x<1$ ) und ein Integral von $x$ bis 1 , wobei er im letzteren wieder $t \mapsto 1-t$ substituiert. Das gibt

$$
s=-\int_{0}^{x} \frac{\ln t}{1-t} \mathrm{~d} t-\int_{0}^{y} \frac{\ln (1-t)}{t} \mathrm{~d} t, \quad y=1-x .
$$

Partielle Integration im ersten Integral und Taylor Entwicklung von $\ln (1-t)$ liefert dann ${ }^{2}$

$$
s=\ln x \ln (1-x)+\sum_{\nu=1}^{\infty} \frac{x^{\nu}+y^{\nu}}{v^{2}} .
$$

Um die Konvergenzgeschwindigkeit der letzten Reihe zu maximieren, nimmt Euler $x=$ $1 / 2$, also $y=1 / 2$, und bekommt

$$
s=(\ln 2)^{2}+\sum_{\nu=1}^{\infty} \frac{1}{2^{v-1} v^{2}} .
$$

Wie man sieht, gelang es Euler, einen Faktor $2^{-v}$ in die Basler Reihe einzuschmuggeln. Die Reihe in (3) konvergiert daher erheblich schneller als die ursprüngliche Reihe in (2). In der Tat, nimmt man $n$ Glieder der Reihe und bezeichnet die resultierende Approximation von $s$ mit $s^{(n)}$, so hat man das in Tabelle 1 gezeigte Konvergenzverhalten:

\footnotetext{
${ }^{1}$ Wir fügen den Arbeiten von Euler deren Eneström-Index Zahlen (E-Zahlen) bei, sowie den Band der Opera omnia, in dem sie zu finden sind, wo OI,14, z.B. Opera omnia, Serie I, Vol. 14 bedeutet. Siehe die Web Seite http: / / www. math. dartmouth. edu/ euler des U.S. Euler Archivs für eine nach den E-Zahlen geordnete kommentierte Liste sämtlicher Werke von Euler.

${ }^{2}$ Hier folgen wir Eulers Vorgehen in $\$ 196$ der Institutiones calculi integralis, Vol. 1, E342, OI,11, und nicht der etwas umständlicheren Herleitung in der zitierten Abhandlung.
} 


\begin{tabular}{rcc}
$n$ & $s^{(n)}$ & Fehler \\
\hline 5 & 1.643543291695979 & $1.39 \times 10^{-03}$ \\
10 & 1.644920051673697 & $1.40 \times 10^{-05}$ \\
20 & 1.644934062865116 & $3.98 \times 10^{-09}$ \\
40 & 1.644934066848226 & $8.88 \times 10^{-16}$
\end{tabular}

Tabelle 1: Konvergenzverhalten der Reihe in (3)

Euler benutzt die Formel (3), um $s$ auf sechs Dezimalstellen zu berechnen.

\section{Eine zweite Approximation}

Der Ausgangspunkt hier ist die bekannte Trapezregel für die Integration einer Funktion $f$,

$$
\int_{1}^{n+1} f(x) \mathrm{d} x \approx \frac{1}{2} f(1)+f(2)+\cdots+f(n)+\frac{1}{2} f(n+1)
$$

die Euler, wie Gregory schon vor ihm, verfeinert indem er auf der linken Seite die Korrekturglieder

$$
\frac{1}{12}[f(n+2)-f(n+1)]-\frac{1}{12}[f(2)-f(1)]
$$

hinzufügt. Man erhält so, nach einfacher Umordnung,

$$
\sum_{\nu=1}^{n+1} f(\nu) \approx \int_{1}^{n+1} f(x) \mathrm{d} x+\frac{1}{12}[5 f(n+1)+f(n+2)]+\frac{1}{12}[7 f(1)-f(2)] .
$$

Nimmt man an, dass $f$ im Unendlichen verschwindet und ins Unendliche summiert und integriert werden kann, so bekommt man, wenn $n \rightarrow \infty$,

$$
\sum_{\nu=1}^{\infty} f(\nu) \approx \int_{1}^{\infty} f(x) \mathrm{d} x+\frac{1}{12}[7 f(1)-f(2)]
$$

Mit Bezug auf das Basler Problem hat Euler in $\$ 14$ von Methodus universalis serierum convergentium summas quam proxime inveniendi (Eine allgemeine Methode, Approximationen zu Summen konvergenter Reihen zu finden, E46; OI,14, S. 101-107; eingereicht 1735, veröffentlicht 1741) nun die sehr nützliche Idee, für ein bestimmtes $v_{0}>1$ die ersten $v_{0}$ Glieder der Reihe direkt zu summieren,

$$
\sum_{\nu=1}^{v_{0}} \frac{1}{v^{2}}=s_{0}
$$

und dann (4) auf $f(x)=\left(v_{0}+x\right)^{-2}$ anzuwenden. Das gibt

$$
s \approx s_{0}+\frac{1}{v_{0}+1}+\frac{1}{12}\left[\frac{7}{\left(v_{0}+1\right)^{2}}-\frac{1}{\left(v_{0}+2\right)^{2}}\right] .
$$


Die Resultate für verschiedene Wahlen von $v_{0}$ sind in Tabelle 2 zusammengestellt:

\begin{tabular}{rcc}
$\nu_{0}$ & $s \approx$ & Fehler \\
\hline 10 & 1.644919055011046 & $1.50 \times 10^{-05}$ \\
20 & 1.644932866546282 & $1.20 \times 10^{-06}$ \\
40 & 1.644933981455983 & $8.54 \times 10^{-08}$ \\
80 & 1.644934061144287 & $5.70 \times 10^{-09}$ \\
160 & 1.644934066479512 & $3.69 \times 10^{-10}$
\end{tabular}

Euler wählte $v_{0}=10$ und erhielt $s \approx 1.644920$, wo aber die zwei letzten Ziffern 19 statt 20 heissen sollten. Im Vergleich mit der ersten Approximation $s^{(n)}$ von (3) konvergiert diese zweite bedeutend langsamer, enthält aber den Keim einer wesentlich allgemeineren und wirksameren Methode, die im nächsten Abschnitt beschrieben werden soll.

\section{Die Euler-Maclaurin Summationsformel}

Offensichtlich ging es Euler nicht nur um die Summe aller reziproken Quadrate, sondern viel allgemeiner um irgendeine Funktion $f$ summiert über alle natürlichen Zahlen, $\sum_{\nu=1}^{\infty} f(v)$. Dies führte zu einer seiner frühen Glanzleistungen - heute die EulerMaclaurin Formel genannt, weil auch Maclaurin sie sechs Jahre später, unabhängig von Euler, gefunden hat. Euler gibt sie zuerst ohne Beweis in Methodus generalis summandi progressiones (Eine allgemeine Methode zur Summierung von Reihen, E25; OI,14, S. 42-72; eingereicht 1732, veröffentlicht 1738) an, und leitet sie in Inventio summae cuiusque seriei ex dato termino generali (Bestimmung der Summe irgend einer Reihe von einem allgemeinen Term, E47; OI,14, S. 108-123; eingereicht 1735, veröffentlicht 1741) vollständig her. In moderner Schreibweise hat sie die Gestalt

$$
\begin{aligned}
\frac{1}{2} f(0)+ & f(1)+\cdots+f(n-1)+\frac{1}{2} f(n) \\
& =\int_{0}^{n} f(x) \mathrm{d} x+\sum_{\mu=1}^{M} \frac{B_{2 \mu}}{(2 \mu) !}\left[f^{(2 \mu-1)}(n)-f^{(2 \mu-1)}(0)\right]+R_{M},
\end{aligned}
$$

wo $B_{2}, B_{4}, B_{6}, \ldots$ die Bernoullischen Zahlen bezeichnen, die Jakob Bernoulli in seiner Ars conjectandi eingeführt hat und durch die Entwicklung

$$
\frac{z}{e^{z}-1}=1-\frac{1}{2} z+\sum_{\mu=1}^{\infty} \frac{B_{2 \mu}}{(2 \mu) !} z^{2 \mu}, \quad|z|<2 \pi,
$$

definiert sind. Euler gibt nie ein Restglied an, aber es kann hier auf verschiedene Art geschrieben werden, z.B. in der Form (vgl. Stoer und Bulirsch [6, §3.3])

$$
R_{M}=\frac{B_{2 M+2}}{(2 M+2) !} \sum_{k=0}^{n-1} f^{(2 M+2)}\left(\xi_{k}\right), \quad k<\xi_{k}<k+1 .
$$

Die Konstanten $B_{2 \mu}$ hat Euler rekursiv berechnet und damals noch nicht als Bernoullische Zahlen erkannt. 
In (7), (8) wird vorausgesetzt, dass die $(2 M+2)$-te Ableitung von $f$ auf $\mathbb{R}_{+}=[0, \infty]$ stetig ist. Nimmt man weiterhin an, dass alle Ableitungen von $f$ ungerader Ordnung bis zur Ordnung $2 M-1$, und $f$ selbst im Unendlichen verschwinden, und $f$ ins Unendliche integrierbar ist, so folgt aus (7), (8), wenn $n \rightarrow \infty$,

$$
\begin{aligned}
\sum_{\nu=1}^{\infty} f(\nu) & =\int_{0}^{\infty} f(x) \mathrm{d} x-\frac{1}{2} f(0)-\sum_{\mu=1}^{M} \frac{B_{2 \mu}}{(2 \mu) !} f^{(2 \mu-1)}(0)+R_{M}, \\
R_{M} & =\frac{B_{2 M+2}}{(2 M+2) !} \sum_{k=0}^{\infty} f^{(2 M+2)}\left(\xi_{k}\right), \quad k<\xi_{k}<k+1 .
\end{aligned}
$$

Die unendliche Reihe in (10) konvergiert unter der Voraussetzung, dass $f^{(2 M+2)}$ auf $\mathbb{R}_{+}$ positiv und monoton abnehmend ist, und auch $f^{(2 M+1)}$ im Unendlichen verschwindet,

$$
f^{(2 M+2)}(x)>0, \quad f^{(2 M+3)}(x)<0, \quad x \in \mathbb{R}_{+} ; \quad f^{(2 M+1)}(\infty)=0 .
$$

Dann gilt nämlich

$$
\begin{gathered}
0<\sum_{k=0}^{n-1} f^{(2 M+2)}\left(\xi_{k}\right)<\sum_{k=0}^{n-1} f^{(2 M+2)}(k)=f^{(2 M+2)}(0)+\sum_{k=1}^{n-1} f^{(2 M+2)}(k) \\
<f^{(2 M+2)}(0)+\int_{0}^{n-1} f^{(2 M+2)}(x) \mathrm{d} x=f^{(2 M+2)}(0)+f^{(2 M+1)}(n-1)-f^{(2 M+1)}(0),
\end{gathered}
$$

und daher, wenn $n \rightarrow \infty$,

$$
0<\sum_{k=0}^{n-1} f^{(2 M+2)}\left(\xi_{k}\right)<f^{(2 M+2)}(0)-f^{(2 M+1)}(0) .
$$

Insbesondere muss auch $f^{(2 M+2)}$ im Unendlichen verschwinden, und man zeigt wie oben, dass die fragliche Reihe das Cauchy-Bolzano Konvergenzkriterium erfüllt. Es folgt

$$
\left|R_{M}\right|<\frac{\left|B_{2 M+2}\right|}{(2 M+2) !}\left[f^{(2 M+2)}(0)-f^{(2 M+1)}(0)\right] .
$$

\section{Anwendungen}

In $\S 31-32$ von E47 wendet Euler die Formel (9) (ohne Restglied!) auf das Basler Problem an, und in $\S 25-26$ auch auf die Berechnung der Eulerschen Konstanten.

\subsection{Anwendung auf das Basler Problem}

Wie schon in (5) summiert Euler die ersten $v_{0}(=10)$ Glieder der Basler Reihe direkt,

$$
s=\sum_{\nu=1}^{\infty} \frac{1}{v^{2}}=s_{0}+\sum_{\nu=1}^{\infty} \frac{1}{\left(v_{0}+v\right)^{2}}
$$

und berechnet die Summe der übrigen Glieder durch Anwendung von (9) auf die Funktion

$$
f(x)=\frac{1}{\left(v_{0}+x\right)^{2}} .
$$


Diese erfüllt wegen $f^{(m)}(x)=(-1)^{m}(m+1) !\left(v_{0}+x\right)^{-(m+2)}$ alle in $\S 4$ gemachten Voraussetzungen, so dass (9), (11), auf (13) angewandt, Folgendes liefert:

$$
\begin{aligned}
\sum_{\nu=1}^{\infty} \frac{1}{\left(v_{0}+v\right)^{2}} & =\frac{1}{v_{0}}-\frac{1}{2} \frac{1}{v_{0}^{2}}+\sum_{\mu=1}^{M} \frac{B_{2 \mu}}{v_{0}^{2 \mu+1}}+R_{M}, \\
\left|R_{M}\right| & <\frac{\left|B_{2 M+2}\right|}{v_{0}^{2 M+3}}\left(1+\frac{2 M+3}{v_{0}}\right) .
\end{aligned}
$$

Man sieht, dass das Restglied im absoluten Betrag, bis auf den Faktor $\left(1+(2 M+3) / v_{0}\right)$, kleiner ist als das erste vernachlässigte Glied der Reihe auf der rechten Seite von (14). Letztes ist ja für alternierende (konvergente) Reihen bekannt; hier allerdings haben wir es mit einer divergenten (asymptotischen) Reihe zu tun. Wegen (vgl. z.B. [1, eq 23.1.15])

$$
\frac{2(2 M+2) !}{(2 \pi)^{2 M+2}}<\left|B_{2 M+2}\right|<\frac{2(2 M+2) !}{(2 \pi)^{2 M+2}} \cdot \frac{1}{1-2^{-(2 M+1)}}
$$

gilt auch

$$
\left|R_{M}\right|<\frac{2(2 M+2) !}{\left(2 \pi \nu_{0}\right)^{2 M+2} \nu_{0}}\left(1+\frac{2 M+3}{\nu_{0}}\right) /\left(1-2^{-(2 M+1)}\right),
$$

was für grosse $M$ mit (15) praktisch identisch ist.

Beste Genauigkeit erhält man, wenn $M=M_{\mathrm{opt}}$ so gewählt wird, dass die obere Schranke in (16) am kleinsten ist. Mit Eulers Wahl $v_{0}=10$ findet man

$$
M_{\mathrm{opt}}=30, \quad\left|R_{M_{\mathrm{opt}}}\right|<1.4966 \times 10^{-26} .
$$

Die Euler-Maclaurin Formel (14), zusammen mit (12) für $v_{0}=10$, ermöglicht es also, die Basler Reihe $s$ mindestens auf 26 Dezimalstellen genau zu berechnen. In der Tat findet man (mit 50-stelliger Arithmetik) die Approximation

$$
s \approx 1.64493406684822643647241516562,
$$

mit einem Fehler von $1.030 \times 10^{-27}$. Euler hat vermutlich mit $M=12$ gerechnet $(\mathrm{ob}-$ wohl er (14) nur für $M=7$ explizit ausschreibt) und so $s$ zu 20 Dezimalstellen genau erhalten. Sehr wahrscheinlich hat dieses genaue Resultat ihm die Identifikation mit $\pi^{2} / 6$ nahegelegt.

\subsection{Berechnung der Eulerschen Konstanten}

Die Eulersche Konstante ist durch den Grenzwert

$$
\gamma=\lim _{n \rightarrow \infty}\left(\sum_{\nu=1}^{n} \frac{1}{v}-\ln n\right)
$$


definiert. Wie zuvor summiert man zunächst die ersten $v_{0}(<n)$ Glieder der Reihe direkt,

$$
s_{0}=\sum_{\nu=1}^{\nu_{0}} \frac{1}{v}
$$

und schreibt dann

$$
\sum_{\nu=1}^{n} \frac{1}{v}=s_{0}+s, \quad s=\sum_{\nu=1}^{n-v_{0}} \frac{1}{v_{0}+v}
$$

Auf $s$ kann die Euler-Maclaurin Formel (7) angewandt werden, wo $n$ durch $n-v_{0}$ zu ersetzen ist, und $f$ durch

$$
f(x)=\frac{1}{v_{0}+x}
$$

Man erhält

$$
s=\ln n-\ln v_{0}-\frac{1}{2 v_{0}}+\frac{1}{2 n}+\sum_{\mu=1}^{M} \frac{B_{2 \mu}}{2 \mu}\left(-\frac{1}{n^{2 \mu}}+\frac{1}{v_{0}^{2 \mu}}\right)+R_{M},
$$

und für den Rest, ähnlich wie in $\$ 5.1$,

$$
\left|R_{M}\right|<\frac{2(2 M+2) !}{\left(2 \pi v_{0}\right)^{2 M+2} \nu_{0}}\left[1+\frac{\nu_{0}}{2 M+2}\left(1-\left(\frac{\nu_{0}}{n}\right)^{2 M+2}\right)\right] /\left(1-2^{-(2 M+1)}\right) .
$$

Addiert man $s_{0}-\ln n$ auf beiden Seiten von (18), und lässt $n \rightarrow \infty$, sowohl in (18) als auch in der Abschätzung des Restglieds, so bekommt man

$$
\gamma=s_{0}-\ln v_{0}-\frac{1}{2 \nu_{0}}+\sum_{\mu=1}^{M} \frac{B_{2 \mu}}{2 \mu} \frac{1}{v_{0}^{2 \mu}}+R_{M},
$$

Wo

$$
\left|R_{M}\right|<\frac{2(2 M+2) !}{\left(2 \pi \nu_{0}\right)^{2 M+2} \nu_{0}}\left(1+\frac{\nu_{0}}{2 M+2}\right) /\left(1-2^{-(2 M+1)}\right) .
$$

Für den optimalen Wert von $M$ erhält man wieder $M_{\mathrm{opt}}=30$, und

$$
\left|R_{M_{\mathrm{opt}}}\right|<2.301 \times 10^{-27} \text {. }
$$

Euler berechnete $\gamma$ auf diese Weise, mit $\nu_{0}=10$, zu 16 korrekten Dezimalstellen, wahrscheinlich mit der Wahl $M=7$, hätte aber mit $M=30$ mehr als zehn weitere Dezimalzahlen erhalten können, nämlich

$$
\gamma=.577215664901532860606512089914
$$

mit einem Fehler von $1.688 \times 10^{-28}$. 


\section{Die Eulersche Reihentransformation}

In dem Werk Institutiones calculi differentialis cum eius usu in analysi finitorum ac doctrina serierum (Grundlagen des Differentialkalküls mit Anwendungen auf die endliche Analysis und Reihen, E212; OI,10; veröffentlicht 1755) leitet Euler in Part II, Ch. 1: De transformatione serierum (Über Reihentransformationen), $§ 3$, unter anderem folgende Transformation her,

$$
\sum_{\nu=0}^{\infty} a_{\nu} x^{\nu+1}=\sum_{n=0}^{\infty}\left(\frac{x}{1-x}\right)^{n+1} \Delta^{n} a_{0},
$$

wo $\Delta$ der Differenzenoperator $\Delta a_{v}=a_{v+1}-a_{v}$ bedeutet. Für $x=-1$ geht sie über in

$$
\sum_{\nu=0}^{\infty}(-1)^{v} a_{\nu}=\sum_{n=0}^{\infty} \frac{(-1)^{n}}{2^{n+1}} \Delta^{n} a_{0},
$$

was heute als Eulersche Reihentransformation bekannt ist ${ }^{3}$. Dafür gibt er viele Beispiele, unter anderem auch solche, die divergente Reihen betreffen, z.B. die relativ harmlose Reihe

$$
s=1-1+1-1+1-1 \pm \cdots,
$$

für die $a_{v}=1$, also $\Delta a_{0}=\Delta^{2} a_{0}=\cdots=0$, und daher $s=\frac{1}{2}$ ist. Eine waghalsigere Reihe ist

$$
s=\sum_{\nu=0}^{\infty}(-1)^{\nu}(\nu+1) !,
$$

für die Euler durch geistreiche Manipulationen $s=.4036524077$ findet. Den exakten Wert kann man durch das Exponentialintegral $E_{1}(x)=\int_{x}^{\infty} e^{-t} \mathrm{~d} t / t$ ausdrücken,

$$
s=1-e E_{1}(1)=.4036526376768 \ldots,
$$

woraus man sieht, dass Euler sich in den letzten vier Ziffern seines Resultats geirrt hat.

Ein klassisches Beispiel (bei Euler in op. cit., §11.I) ist die sehr langsam konvergente Reihe

$$
s=\sum_{\nu=0}^{\infty} \frac{(-1)^{v}}{v+1}=\ln 2,
$$

für welche Eulers Transformation die wesentlich schneller konvergierende Reihe

$$
s=\sum_{n=0}^{\infty} \frac{1}{(n+1) 2^{n+1}}
$$

liefert. Etwas interessanter ist die Leibnizsche Reihe (ibid., §11.II)

$$
s=\sum_{\nu=0}^{\infty} \frac{(-1)^{\nu}}{2 v+1}=\frac{\pi}{4},
$$

\footnotetext{
${ }^{3}$ Nach Otto Spiess [5, §5, Fussnote 1] benutzte Euler diese Transformation bereits 1743 in einem Brief an Goldbach
} 
für die $a_{v}=1 /(2 v+1)$ und $\Delta^{n} a_{0}=(-1)^{n} 2^{2 n} n !^{2} /(2 n+1) !$ ist, also

$$
s=\sum_{n=0}^{\infty} \frac{2^{n-1} n !^{2}}{(2 n+1) !} .
$$

Das allgemeine Glied, nach Stirlings Formel, ist für $n \rightarrow \infty$ äquivalent $\sqrt{\frac{\pi}{2 n}} 2^{-(n+1)}$, so dass die Konvergenzbeschleunigung hier etwa gleich gross ist wie im vorherigen Beispiel.

Allgemein kann man sagen, dass (22) gültig ist, falls die Reihe auf der linken Seite von (22) (die nicht notwendigerweise alternierend, also $a_{v}>0$, sein muss) konvergiert. Dann konvergiert auch die Reihe auf der rechten Seite, und zwar zum selben Grenzwert, aber nicht notwendigerweise schneller. Man hat Konvergenzbeschleunigung dann, wenn alle $a_{v}>0$, die Folge $\left\{a_{\nu}\right\}_{\nu=0}^{\infty}$ vollständig monoton, d.h. $(-1)^{n} \Delta^{n} a_{k}>0$ ist für alle $n, k=$ $0,1,2, \ldots$, und $a_{v+1} / a_{v} \geq a>\frac{1}{2}$ gilt. Die Konvergenzbeschleunigung ist in der Tat um so beträchtlicher, je grösser $a$ ist (Knopp [4, Satz 155]; der Operator $\Delta$ ist bei Knopp als rückwärtiger Differenzenoperator definiert, also ist er das Negative unseres Operators).

\section{Die Lambertsche Reihe}

Zum Schluss noch eine kleine Perle aus Eulers Werkzeugkasten für unendliche Reihen, die zwar nichts mit dem Vorhergehenden zu tun hat, aber dennoch einen Einblick gestattet in Eulers Einfallsreichtum. Es handelt sich um die Lambertsche Reihe

$$
s(x)=\sum_{\nu=1}^{\infty} \frac{1}{x^{\nu}-1}, \quad x>1,
$$

speziell für den Fall wo $x=10$, dem Euler im Zusammenhang mit einem missglückten Interpolationsversuch begegnet ist (vgl. [3], wo $s(10)=-S(0)$ ). Die Reihe tritt an verschiedenen Stellen der Arbeit Consideratio quarumdam serierum, quae singularibus proprietatibus sunt praeditae (Betrachtung einiger Reihen, die sich durch spezielle Eigenschaften auszeichnen, E190; OI,14, S. 516-541; eingereicht 1750, veröffentlicht 1753) auf, z.B. in $\S \S 28-29$. Dort entwickelt Euler jedes Glied der Reihe (23) in eine geometrische Reihe in Potenzen von $1 / x$, und sammelt dann alle Glieder mit gleicher Potenz. So erhält er

$$
s=\frac{1}{x}+\frac{2}{x^{2}}+\frac{2}{x^{3}}+\frac{3}{x^{4}}+\frac{2}{x^{5}}+\frac{4}{x^{6}}+\frac{2}{x^{7}}+\frac{4}{x^{8}}+\frac{3}{x^{9}}+\cdots .
$$

Ein Meister im Aufspüren von versteckten regelmässigen Mustern, Euler bemerkt nun, dass der Zähler in jedem Bruch genau gleich der Anzahl der Teiler der entsprechenden Potenz von $1 / x$ ist, also z.B. in $4 / x^{6}$ ist 4 gleich der Anzahl der Teiler 1, 2, 3, 6 von 6. Wenn $x=10$, kann das Resultat mühelos in Dezimalform hingeschrieben werden, was Euler bis auf 30 Stellen tut:

$$
s=.122324243426244526264428344628 \ldots \text {. }
$$

Hier ist die Anzahl der Teiler stets kleiner als 10; wenn sie grösser oder gleich 10 ist, müssen kleine Anpassungen vorgenommen werden. Das ist zum ersten Mal an der 49-ten Dezimalstelle der Fall.

Dank. Für den Vorschlag in Fussnote 2 danke ich dem anonymen Begutachter der Arbeit. 


\section{Literatur}

[1] Abramowitz, M.; Stegun, I.A.: Handbook of mathematical functions with formulas, graphs, and mathematical tables. NBS Appl. Math. Series, vol. 55, Washington, DC, 1964.

[2] Bernoulli, J.: Positiones arithmetica de seriebus infinitis, earumque summa finita. Basel 1689. [Auch in Opera Jacobi Bernoulli, Vol. 1, Geneva 1744, 375-402; esp. 398.]

[3] Gautschi, W.: On Euler's attempt to compute logarithms by interpolation: A commentary to his letter of February 16, 1734 to Daniel Bernoulli. J. Comp. Appl. Math., to appear.

[4] Knopp, K.: Theorie und Anwendung der unendlichen Reihen. 4-te Ed., Springer, Berlin 1947.

[5] Spiess, Otto: Die Summe der reziproken Quadratzahlen. Festschrift zum 60. Geburtstag von Prof. Dr. Andreas Speiser, 66-86. Füssli, Zürich 1945.

[6] Stoer, J.; Bulirsch, R.: Introduction to numerical analysis. Texts in Applied Mathematics, vol. 12, Springer, New York 2002

\section{Walter Gautschi}

Department of Computer Sciences

Purdue University

250 N. University Street

West Lafayette, IN 47907-2066, USA

e-mail:wxg@cs.purdue.edu 